\title{
Interruption of Sneddon-Wilkinson Subcorneal Pustulation with Infliximab
}

\author{
Lorenz Kretschmer $^{\mathrm{a}} \quad$ Julia-Tatjana Maul ${ }^{\mathrm{a}} \quad$ Thomas Hofer $^{\mathrm{b}}$ \\ Alexander A. Navarini ${ }^{a}$ \\ ${ }^{a}$ Department of Dermatology, University Hospital of Zurich, Zurich, Switzerland; \\ ${ }^{b}$ Praxisgemeinschaft Winkelried, Wettingen, Switzerland
}

\section{Keywords}

Infliximab · Sneddon-Wilkinson disease · Subcorneal pustular dermatosis - TNF- $\alpha$ antagonist

\begin{abstract}
Subcorneal pustular dermatosis (SCPD, Sneddon-Wilkinson disease) is a rare chronicrelapsing skin disorder that typically manifests as flaccid sterile pustules without systemic symptoms. Although the accumulation of neutrophils is acknowledged to be a hallmark of SCPD, its exact pathomechanism is still not known. Several chemotactic factors have been implicated in neutrophil recruitment and invasion, including the proinflammatory cytokine TNF- $\alpha$. These findings correspond well with clinical reports of successful off-label use of TNF blocking agents in cases that were refractory to first-line therapy, mostly with dapsone. We report the case of a 29-year-old male with atypical and severe manifestation of SCPD that resolved after a single dose of infliximab. Consolidation was observed 1 day after treatment and regression of skin lesions occurred after a few days. Residual scarring and postlesional hyperpigmentation was seen at a 2-month follow-up appointment. The patient was initiated on a daily maintenance therapy with dapsone, which led to a drop in hemoglobin and had to be stopped. Upon development of small, scaly lesions, a maintenance therapy with infliximab was started and the patient has had no recurrence to date. Anti-TNF agents present a promising option for patients affected by severe SCPD. We review the reports of similar cases in the literature to date.

(C) 2017 The Author(s)

Published by S. Karger AG, Basel
\end{abstract}




\section{Case Reports in Dermatology}

\section{Introduction}

Subcorneal pustular dermatosis (SCPD) is a rare chronic-relapsing skin disorder that was first described by Sneddon and Wilkinson [1] in 1956. The characteristic primary skin lesions are pustules, which can develop into flaccid blisters, with hypopyon-like accumulation of pus in their lower part and clear fluid in their upper part. Fresh pustules measure several millimeters in diameter and can coalesce into annular patterns. Ruptured blisters can result in superficial scaling, crusting, and secondary hyperpigmentation. Typical localizations involve the trunk, intertriginous areas, and flexural sites of the extremities [2]. The characteristic histological finding of SCPD involves a superficial split below the stratum corneum, with an abundance of neutrophils in the blister but without spongiosis or acantholysis of the epidermis. In addition, a mixed perivascular inflammatory infiltrate is found in the upper dermis. Immunofluorescence is classically negative [3]. The accumulation of neutrophils is nowadays recognized as an important mediator of SCPD [4, 5], but its underlying causes remain insufficiently understood. However, a role for TNF has been implicated. Elevated cytokine levels have been reported in serum and blister fluids [6], which might drive neutrophil activation and accumulation [7, 8]. In addition, several clinical reports have described effective off-label therapy using TNF blocking agents in cases that responded insufficiently to first-line therapy, such as with dapsone $[7,9]$.

\section{Case Report}

A 29-year-old male IT specialist presented with a 3-week history of severe, progressive pustular skin eruptions. The lesions had begun as a maculopapular rash on the patient's axillae 1 day after he had visited a thermal bath, and had spread over the course of the next days. Previous therapy with topical and high-dose oral corticosteroids, as well as coamoxicillin, had been ineffective. Upon initial evaluation at our department, the patient exhibited plaques and large flaccid blisters that covered most of the patient's trunk (Fig. 1a) but also the face and intertriginous areas. Some of the blisters showed hypopyon-like accumulation of pus in their lower part and clear fluid in their upper part. Ruptured blisters displayed crusting and scales around the edges of the lesions. Mucous membranes were not affected. The patient suffered from pruritus; however, fever and other symptoms of systemic disease were not present. Due to the severity of the patient's skin affection and the associated risk of septic complications, he was admitted to hospital for further evaluation and treatment. Virology testing for herpes simplex virus infection was negative. Bacterial cultures obtained from intact blisters showed an abundance of $\beta$-hemolytic Streptococci and Staphylococcus aureus species. Thus, an (albeit very severe) bullous impetigo was suspected and treated with potassium permanganate containing baths and daily intravenously amoxicillinclavulanate potassium (Augmentin), in accordance with antibiotic susceptibility testing. In addition, topical corticosteroid treatment and the antipruritic agent polidocanol were administered. However, bacterial cultures obtained aseptically from the bottom of newly punctured blisters were negative. At best, marginal improvement was found as a result of 2 courses of amoxicillin in total (oral, then intravenously) for 13 days (Fig. 1b). Direct immunofluorescence on skin specimen from primary lesions was negative, as was a serological screen for antibodies directed against skin antigens, including desmoglein 1 and 3. Histopathology revealed subcorneal pustules, with only mild acantholysis and no spongiosis of the underlying epidermis (Fig. 1c). A revision of all results led us to consider the diagnosis of 
SCPD. Additional tests were performed to screen the patient for systemic diseases that have previously been associated with SCPD, namely IgA or IgG gammopathy and multiple myelo$\mathrm{ma}$, but these were inconclusive. A single dose of $350 \mathrm{mg}$ infliximab was administered. Consolidation was observed 1 day after treatment and regression of skin lesions occurred after a few days (Fig. 1d). Residual scarring and postlesional hyperpigmentation was seen at a 2month follow-up appointment (Fig. 1e). No side effects were noted in response to infliximab. A daily maintenance therapy with dapsone was initiated, which led to a drop in hemoglobin and had to be stopped after 2 months. Upon development of small, scaly lesions, a maintenance therapy with infliximab was started thereafter. Since then, the patient has remained free of relapses.

\section{Discussion}

SCPD is a rare skin disorder, which typically presents in women over the age of 40 [2]. Some cases in children and adolescents have been reported, but several displayed atypical features [3]. Accompanying systemic symptoms or a history of other skin conditions might complicate the diagnosis. In particular, the differentiation between SCPD and pustular psoriasis can be very challenging [10]. Furthermore, secondary infection with Streptococci or $S$. aureus species is possible [3], which might complicate the initial distinction of SCPD from bullous impetigo [11]. It has further been debated whether SCPD is an independent disease entity or rather represents a subentity of other diseases [12]. An overlap with IgA pemphigus has been described [4], which has been linked to the autoantigen desmocollin 1 [13]. Although the course of SCPD is generally considered to be benign, it can be associated with several systemic diseases. Notably, IgA or IgG gammopathy and other lymphoproliferative disorders, especially multiple myeloma, have been observed $[3,14]$. These findings might indicate a certain predisposition for immune dysregulation. Although the subcorneal accumulation of neutrophils appears to be a hallmark of SCPD, its exact pathomechanism is still not known. Several chemotactic factors have been implicated in neutrophil recruitment and invasion, including the proinflammatory cytokine TNF [6]. These findings correspond well with clinical reports of successful off-label use of TNF blocking agents in cases that were refractory to first-line therapy, mostly with dapsone [7, 9]. Complete responses to infliximab were observed in 2 cases $[15,16]$. Other reports describe initially good responses to infliximab, which were gradually lost with repeated infusions of infliximab [17] or lost due to relapse [18]. Complete responses to etanercept were observed in 3 cases, 1 of which received a combination with low-dose acitretin $[19,20]$, and an additional report describes a near-tocomplete response [21]. In 1 case, a complete response to adalimumab could be observed [22]. Following the loss of efficacy of an anti-TNF agent, switching to a different agent has been successful. One case describes the stepwise loss of efficacy of infliximab, and then etanercept, but clearance under adalimumab [23].

Considering these cases reported in the literature to date and the severity of manifestation observed in this case, the patient responded exceptionally well to infliximab treatment. With a single dose, the drug interrupted the pustulation, demonstrating the power of targeted therapies in rare neutrophil-mediated diseases. 


\section{Statement of Ethics}

The authors have no ethical conflicts to disclose.

\section{Disclosure Statement}

The authors declare no conflicts of interest and no financial interests.

\section{References}

1 Sneddon IB, Wilkinson DS: Subcorneal pustular dermatosis. Br J Dermatol 1956;68:385-394.

-2 Cheng S, Edmonds E, Ben-Gashir M, Yu RC: Subcorneal pustular dermatosis: 50 years on. Clin Exp Dermatol 2008;33:229-233.

3 Reed J, Wilkinson J: Subcorneal pustular dermatosis. Clin Dermatol 2000;18:301-313.

-4 Navarini AA, Satoh TK, French LE: Neutrophilic dermatoses and autoinflammatory diseases with skin involvement-innate immune disorders. Semin Immunopathol 2016;38:45-56.

5 Prat L, Bouaziz JD, Wallach D, Vignon-Pennamen MD, Bagot M: Neutrophilic dermatoses as systemic diseases. Clin Dermatol 2014;32:376-388.

6 Grob JJ, Mege JL, Capo C, Jancovicci E, Fournerie JR, Bongrand P, Bonerandi JJ: Role of tumor necrosis factor-alpha in Sneddon-Wilkinson subcorneal pustular dermatosis. A model of neutrophil priming in vivo. J Am Acad Dermatol 1991;25:944-947.

7 Patel R, Cafardi JM, Patel N, Sami N, Cafardi JA: Tumor necrosis factor biologics beyond psoriasis in dermatology. Expert Opin Biol Ther 2011;11:1341-1359.

-8 Shalaby MR, Palladino MA Jr, Hirabayashi SE, Eessalu TE, Lewis GD, Shepard HM, Aggarwal BB: Receptor binding and activation of polymorphonuclear neutrophils by tumor necrosis factor-alpha. J Leukoc Biol 1987;41:196-204.

-9 Alexis AF, Strober BE: Off-label dermatologic uses of anti-TNF-a therapies. J Cutan Med Surg 2005;9:296-302.

-10 Limmer BL: Letter: Subcorneal pustular dermatosis vs pustular psoriasis. Arch Dermatol 1974;110:131.

-11 Speeckaert MM, Speeckaert R, Lambert J, Brochez L: Acute generalized exanthematous pustulosis: an overview of the clinical, immunological and diagnostic concepts. Eur J Dermatol 2010;20:425-433. Pinkus H: Is subcorneal pustular dermatosis of Sneddon and Wilkinson and entity sui generis? Am J Dermatopathol 1981;3:379-380.

13 Hashimoto T, Kiyokawa C, Mori O, Miyasato M, Chidgey MA, Garrod DR, Kobayashi Y, Komori K, Ishii K, Amagai M, Nishikawa T: Human desmocollin 1 (Dsc1) is an autoantigen for the subcorneal pustular dermatosis type of IgA pemphigus. J Invest Dermatol 1997;109:127-131.

14 Kasha EE Jr, Epinette WW: Subcorneal pustular dermatosis (Sneddon-Wilkinson disease) in association with a monoclonal IgA gammopathy: a report and review of the literature. J Am Acad Dermatol 1988;19:854-858.

15 Voigtlander C, Luftl M, Schuler G, Hertl M: Infliximab (anti-tumor necrosis factor alpha antibody): a novel, highly effective treatment of recalcitrant subcorneal pustular dermatosis (Sneddon-Wilkinson disease). Arch Dermatol 2001;137:1571-1574.

16 Naretto C, Baldovino S, Rossi E, Spriano M, Roccatello D: The case of SLE-associated Sneddon-Wilkinson pustular disease successfully and safely treated with infliximab. Lupus 2009;18:856-857.

17 Bonifati C, Trento E, Cordiali Fei P, Muscardin L, Amantea A, Carducci M: Early but not lasting improvement of recalcitrant subcorneal pustular dermatosis (Sneddon-Wilkinson disease) after infliximab therapy: relationships with variations in cytokine levels in suction blister fluids. Clin Exp Dermatol 2005;30:662-665.

18 Raimondo N, Gonzalez L, Orden A, Carvalho A: Sneddon Wilkinson syndrome: a therapheutic challenge. Poster abstract presented at the American Academy of Dermatology 65th Annual Meeting. J Am Acad Dermatol 2007;56(suppl 2):AB46.

19 Berk DR, Hurt MA, Mann C, Sheinbein D: Sneddon-Wilkinson disease treated with etanercept: report of two cases. Clin Exp Dermatol 2009;34:347-351.

20 Iobst W, Ingraham K: Sneddon-Wilkinson disease in a patient with rheumatoid arthritis. Arthritis Rheum 2005;52:3771.

21 Bedi MK: Successful treatment of long-standing, recalcitrant subcorneal pustular dermatosis with etanercept. Skinmed 2007;6:245-247. 


\section{Case Reports in Dermatology}

\begin{tabular}{l|l}
\hline Case Rep Dermatol 2017;9:140-144 \\
\hline DOI: $10.1159 / 000468917$ & $\begin{array}{l}\text { (c) 2017 The Author(s). Published by S. Karger AG, Basel } \\
\text { www.karger.com/cde }\end{array}$ \\
\hline
\end{tabular}

Kretschmer et al.: Interruption of Sneddon-Wilkinson Subcorneal Pustulation with Infliximab

22 de Encarnacao Roque Diamantino F, Dias Coelho JM, Macedo Ferreira AM, Fidalgo AI: Subcorneal pustular dermatosis treated successfully with adalimumab. Eur J Dermatol 2010;20:512-514.

-23 Versini M, Mantoux F, Angeli K, Passeron T, Lacour J-P: Maladie de Sneddon-Wilkinson échappant à l'infliximab et à l'étanercept: efficacité de l'adalimumab. Ann Dermatol Venereol 2013;140:797-800.
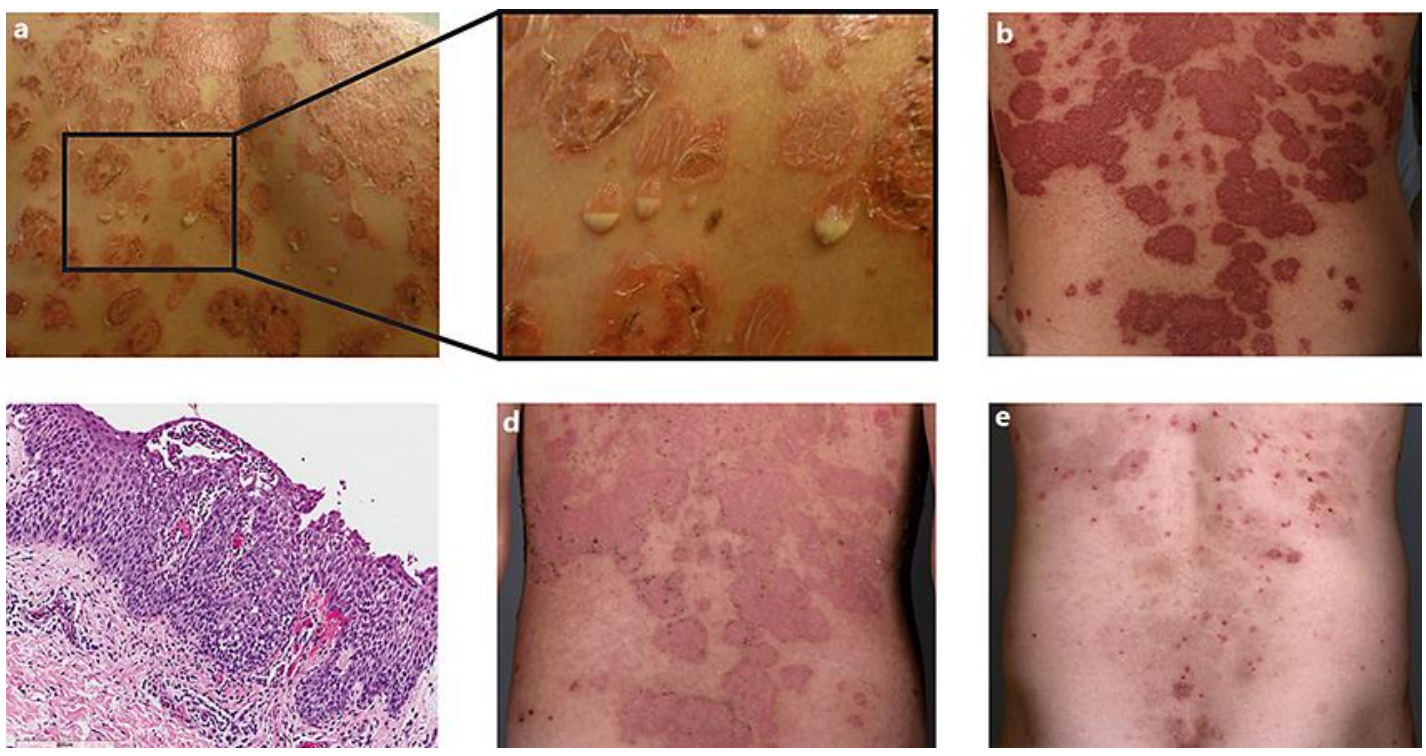

Fig. 1. a Disseminated, sharply-demarcated erythematous plaques and hypopyon-like blisters on the patient's upper trunk upon initial evaluation at the outpatient department. b At best, marginal improvement was found after amoxicillin treatment over 13 days. c Histopathology of specimen from a skin lesion showing a subcorneal pustule, with only mild acantholysis and no spongiosis of the underlying epidermis. Hematoxylin and eosin, $\times 12$. d Regression of skin lesions 4 days after treatment with infliximab. e Residual scarring and postlesional hyperpigmentation 2 months after treatment with infliximab. 